\title{
Material handling robots fleet size optimization by a heuristic
}

\author{
V. K. Chawla ${ }^{a *}$, A. K. Chanda ${ }^{b}$ and Surjit Angra ${ }^{c}$
}

${ }^{a}$ Department of Mechanical and Automation Engineering, Indira Gandhi Delhi Technical University for Women, Delhi, India ${ }^{b}$ Department of Mechanical and Automation Engineering, G.B. Pant Engineering College, Delhi, India c Department of Mechanical Engineering, National Institute of Technology, Kurukshetra, Haryana, India CH RON I C L E

\section{Article history:}

Received: March 82019

Received in revised format:

April 22019

Accepted: April 22019

Available online:

April 42019

Keywords:

Fleet size optimization

Material handling robots

Modified memetic particle

swarm optimization algorithm

\section{A B S T R A C T}

The application of material handling robots (MHRs) has been commonly observed in flexible manufacturing systems (FMS) for efficient material handling activities. In order to gain maximum throughput, minimum tardiness from the minimum investment of funds for the material handling activities, it is important to determine the optimum numbers of MHRs required for efficient production of jobs in the FMS. In the present work, the requirement of MHRs is optimized for different FMS layouts by using a heuristic procedure. Initially, a mathematical model is proposed to identify the MHRs requirement to perform the material handling activities in the FMS, later on, the model is optimized by simulating a novel heuristic procedure to find the required optimum number of MHRs in the FMS. The proposed methodology is found to be generic enough and can also be applied in various industries employing the MHRs.

\section{Introduction}

The flexible manufacturing system (FMS) consists of flexible, reprogrammable, highly advanced and accurate computer-controlled manufacturing systems and accessories. The sustainable FMS operations can be realized by keeping minimum waiting time or idle time of manufacturing centers and by yielding optimum utilization of other manufacturing resources of FMS, simultaneously (Angra et al., 2018; Chanda et al., 2018; Chawla et al., 2017; 2018a 2018b; 2018c; 2018d; 2018e; 2018f; 2018g; 2019). The optimum utilization of FMS resource assures high throughput with minimum makespan. The material handling robot (MHR) is used in the FMS facility for material handling operations. MHRs are capable of load /unload and transfer jobs in semi-finished or in finished condition between the work centers and other designated points in the FMS facility. The application of MHRs for material handling operations can also be commonly observed in a number of automated commercial industries such as flexible manufacturing facilities, semiconductor industries, sea-port container terminals, automobile industries, etc. The association of FMS and MHRs can yield a significant increase in the throughput for entire FMS. With a high degree of control and 
flexibility, the performance of MHRs is found to be remarkable compared with other conventional material handling equipment applied for manufacturing operations. From the research of Tompkins and White (1984), it is evident that $20-50 \%$ of operations' expenditure are incurred only in the material handling activities hence optimum allocation and utilization of material handling resources are of paramount importance for savings in overall operational cost of any manufacturing plant. In real time manufacturing conditions, the scheduling and dispatching of MHRs is found to be complex and critical therefore under real time manufacturing conditions the resources are found to be exceeding than their actual requirements and sometimes the resources are found to be insufficient. To avoid over and insufficient investment of funds in FMS resources the estimation and selection of the optimum quantity of resources becomes highly significant. The estimation and optimization of MHRs fleet size in FMS is one of the significant steps for optimum utilization of resources and invested funds in the FMS facility (Mahadevan \& Narendran, 1990; Ganesharajah et al., 1998). In this paper, optimization of MHRs fleet size to be deployed in different sizes of FMS layout is carried out. Initially, the problem is solved analytically and later on the results are further optimized by using a novel heuristic procedure. The applied methodology of optimization is found to be generic and can also be applied effectively to different sizes of FMS layouts. In the present study, various variables namely job sequence, job mix, processing time, job flow, a loading-unloading station and number of work centers are considered in the analytical model and further for optimization of MHRs fleet size by the MMPSO algorithm.

The appropriate selection of MHR's (also referred as automatic guided vehicles (AGVs)) fleet size for material handling activities in the FMS is one of the most common and basic steps in the installation of the FMS facilities. The optimum fleet size of MHRs or automatic guided vehicle (MHRs) can significantly increase the throughput of the FMS (Van der Meer, 2000). The deployment of MHRs for different material handling activities requires considerable investments of funds. In order to yield a good return on the invested capital, optimum utilization of MHRs in material handling activities for the FMS becomes highly significant. Moreover, the optimum deployment of MHRs fleet size also leads to the reduction in wastage of resources, reinforce sustainability and increase the overall utility of resources. The tandem FMS facilities are divided into several zones and each zone of tandem FMS layout is served by a dedicated MHR which is observed to be sufficient for fulfilling zonal material handling requirements (Fan et al., 2015). In other FMS layouts, the number of MHRs can vary according to the requirement of the material handling operations, hence appropriate estimation of MHR fleet size becomes crucial to avoid wastage of resources in the overall production operations. The three main factors affecting the estimation of MHR fleet size in FMS namely (1) job transfer points (2) guide path layout (3) MHR dispatching rules or scheduling rules were identified by Egbelu and Tanchoco (1986). The authors applied four analytical models for the estimation of MHRs fleet size and analyzed the MHR fleet size on the basis of the number of trips carried out by a loaded MHR between various machining centers in the manufacturing facility. Authors also pointed out the importance of scheduling and dispatching rules for the appropriate estimate and deployment of the MHRs fleet size. Maxwell and Muckstadt (1982) and Mahadevan and Narendran (1994) performed similar research and estimated MHR fleet size for the FMS facility. The scheduling and dispatching policies also have a pivotal role in the appropriate estimation of MHRs requirements. Srinivasan et al. (1994) investigated a modified first in first out (MFIFO) dispatching rule for the multi-load AGVs serving in the FMS facility. Authors evaluated K time's velocity for the K number of AGVs in the FMS facility and found that the fewer number of AGVs yield a satisfactory result and contrary the quality of solution deteriorate with the deployment of a large number of AGVs. A queuing model for AGVs fleet size estimation was proposed by Tanchoco et al. (1987). Another technique for approximation of the necessary number of AGVs was proposed by Arifin and Egbelu (2000). Vis et al. (2001) implemented a network flow technique for AGV fleet size approximation and developed a polynomial time minimum-flow algorithm for the appropriate calculation of the required number of AGVs. Sinriech and Tanchoco (1992) investigated segmented flow topology (SFT) layout for the FMS facility and proposed an MCDM technique for the optimization of MHRs fleet size. A simultaneous analytical and simulation procedure for estimation 
of MHRs fleet size for the FMS facility was proposed by Yifei et al. (2010), in the proposed model the output of the analytical model was considered as the input of the simulation model. Ji and Xia (2010) proposed an analytical model with an objective to guarantee the transportation system's stability and also to minimize the MHR fleet size. Lin et al. (2010) considered uncertainties in a semiconductor manufacturing industry and developed a simulation-optimization model to find out the optimum fleet size of the MHRs. Authors validated their model by an empirical study. Moghaddam et al. $(2010,2012)$ proposed the robust optimization solutions for the real-time vehicle routing problem for minimizing the uncertain requirements for medicine distribution. In order to analyze and validate the effect of robustness and trade-offs, the computational experiments were carried out. Choobineh et al. (2012) proposed an analytical model for estimation of MHR's fleet size under the steady-state conditions after considering loaded and empty-travel times; MHR's loading - unloading time and waiting time for dispatch of MHRs and validated their methodology by comparing results with the solution yield of simulation methods. Huang et al. (2012) optimized MHRs allocation for a $300 \mathrm{~mm}$ wafer fab industry by the application of discrete event simulation experiment. Chang et al. (2014) proposed a procedure for optimizing the fleet size of MHRs by application of the simulation sequential modeling after considering the minimum vehicle cost under the time constraint and validated the simulation sequential modeling approach by developing few metamodels on the real-time data.

Vivaldini et al. (2016) estimated the minimum number of MHRs requirement for the execution of a specific transportation order within a specific time window. Singh and Khan (2016) proposed an analytical model with minimum computational time for the solution of the loading and unloading problem in the FMS after considering the machine processing time as a primary input. Chawla et al. (2018a, 2018b and 2018d, 2019) developed optimized solutions for the simultaneous scheduling of MHRs and optimized the MHRs fleet size by the application of the modified memetic particle swarm optimization (MMPSO) algorithm, clonal selection (CS) algorithm and grey wolf optimization (GWO) algorithm. Angra et al. (2018) evaluated the performance of different priority dispatching rules when applied to multi-load MHRs in variable sized FMS configurations.

From the literature review, it is observed that the MHR fleet size estimation is carried out in concurrence with appropriate scheduling and dispatching rules, vehicle congestion conditions, FMS facility layout, and traffic management, etc. but a generic procedure to optimize the MHR fleet size in the FMS facility cannot be found in the literature. So a potential research gap is observed for the estimation and further optimization of MHRs fleet size in the FMS by application of the MMPSO algorithm. The present study attempts to fill the aforesaid research gap. In the present study initially, an analytical model for estimation of MHRs fleet size is considered and further optimization of MHRs fleet size is carried out by the application of MMPSO algorithm.

\section{Problem Definition}

The optimization of the manufacturing system's resources to obtain maximum throughput always has been a matter of prime interest. An optimum deployment of resources in a manufacturing system and their utilization delivers productivity with a balanced load on all the resources. An optimum estimate of MHRs fleet size for material handling activities in the FMS is highly essential to reduce the initial capital investment and increase the throughput of the FMS facility (Mahadevan \& Narendran, 1990). In this study, various generic factors for a FMS such as the number of jobs, job processing sequence, number of the work centers, total time consumption, job volume mix, job production time, average time etc. are analyzed in the analytical model for the calculation of the minimum number of MHRs requirement in the different sizes of the FMS layouts. The result yields of the analytical model are optimized by using the modified memetic particle swarm optimization (MMPSO) algorithm in the Matlab software for the different sizes of the FMS facility. 


\subsection{Assumptions}

1. Entry and exit of the job into the manufacturing system is only through load-unload station only.

2. There can be more than one processing sequence for a job.

3. The pick-up and drop-off travel time are deterministic.

4. The average delivery rate of work center is known. If no pick-up and drop-off request are identified then MHR will wait at its on-going position till the new request is generated and received.

5. The number of work centers, type of FMS layout, buffer details, track information and cruising time are known.

6. If more than one idle MHR waits for pick-up and drop-off request then any idle MHR will take pick-up and drop-off request according to the predefined dispatching rule. If more than one pick-up and drop-off request are waiting for idle MHR, then the pick-up and drop-off requests are considered according to the first in first out (FIFO) dispatching rule.

7. The job set-up time is negligible.

\subsection{Estimation of MHRs fleet size - The analytical model}

A job enters into the manufacturing system from the loading and unloading center. The average rate of job entry into the manufacturing system is calculated from the average time spent by the jobs for their productions in the manufacturing system. Meantime consumed by the job is calculated by multiplication of total operation time of the job and the job's probability from the probability matrix. The minimum number of MHRs required in the FMS facility is calculated by the division of each job's mean time with the total available time with the job. Initially, the MHR fleet size is calculated analytically (referring to Eq. (1) to Eq. (9)). The following variables are considered in the equations: the sequence of job processing, the number of work centers, the volume of job mix, and the time consumed in the processing of jobs, total available time and the average time consumed by the jobs, etc.

Notations:

$n=$ number of jobs to be produced.

$v_{i}=$ the volume of job mix for the job $i$.

$N S E Q_{i}=$ the number of production operations required in the production of job $i$.

$S_{i j}=$ the probability of production of job $i$ according to the $j_{\text {th }}$ sequence.

$N M A C_{i j}=$ the number of work centers job $i$ visit for completion of $j_{t h}$ sequence.

$P T_{i j k}=$ the processing time for job $i$ on $k_{t h}$ work center processed according to the $j_{t h}$ sequence.

$t_{k l}=$ the time consumed in travel between two points $k$ and $l$.

In the FMS facility, the load-unload center is considered as $m+1$ work center, the job flow $\left(f_{k l}\right)$ between any two work centers $k$ and $l$, and is given by:

$$
\begin{gathered}
f_{k l}=\sum_{i=1}^{n} v_{i} \sum_{j=1}^{N S E Q_{i}} S_{i j} \delta_{k l} \quad \forall k, l=1, \cdots, m+1 \text { and } \quad k \neq 1 \\
\delta_{k l}=\left\{\begin{array}{l}
1 \text { if work center } l_{t h} \text { is visited just after } k \text { in the } j t h \\
\text { sequence of the job } i, 0 \text { otherwise. }
\end{array}\right\} \\
\mu=\text { the property for flow matrix. } \\
f_{k k}=0 \quad \forall k=1, \ldots, m+1
\end{gathered}
$$


$\sum_{k=1}^{m} f_{m+1, k}=1$

$\sum_{l=1}^{m} f_{l, m+1}=1$

$\sum_{l=1}^{m+1} f_{k l}=\sum_{l=1}^{m+1} f_{l k} \quad \forall k=1, \cdots, m+1$

$\left\{\begin{array}{l}1=\text { if job visits work centre } l . \\ 0=\text { if job does not visit any work centre. }\end{array}\right\}$

$n \operatorname{load} k=480 / \mu_{k}$

$f_{k l}=\sum_{i=1}^{n} v_{i} \sum_{j=1}^{N S E Q_{i}} \sum_{l=1}^{N M A C_{i j}}\left[\frac{\left\{v_{i} S_{i j} \gamma_{k(i j)}\right\}}{\sum_{i=1}^{n} \sum_{j=1}^{N S E Q_{i}} v_{i} S_{i j} \gamma_{k(i j)}}\right] \times P T_{i j k} \quad \forall k=1, \cdots, m$

$$
\begin{aligned}
& \mathrm{TR}=\alpha *\left\{\sum_{k=1}^{m+1} n \operatorname{load}_{k} *\left[\sum_{k=1}^{m+1} p_{k l}\left(t_{k b}+t_{k l}+l_{k}+u_{b}+l_{b}+u_{l}\right)\right]\right\}+(1-\alpha) *\left\{\sum_{k=1}^{m+1} n \operatorname{load}_{k} *\left[\sum_{k=1}^{m+1} p_{k l}\left(t_{k l}+l_{k}+u_{l}\right)\right]\right\} \\
& N=\frac{T R}{T A}
\end{aligned}
$$

The probability matrix for transition $P=\left[P_{k}\right]$ is given by solving the $F$ process matrix $F$ so that the sum of the row is 1 means the probability always remains between 0 and 1 .

$\mu_{k}=$ the average rate at which work center $k$ processes the jobs.

Where

$$
\gamma_{k(i j)}=\left\{\begin{array}{l}
1 \text { if machine } k \text { is visited in the } j \text { th sequence of job } i \\
0 \text { otherwise }
\end{array}\right\}
$$

Let the $=$ number of times jobs are sent via a centralized buffer in case of less storage in front of the work centers.

where,

$T R=$ Total time available for all MHRs.

$T A=$ Time available per MHR.

The constraint of less storage area in front of the work centers is satisfied by routing the jobs through the central buffer. Let $\alpha$ be the time happened hence the total time for all MHRs $(T R)$ is calculated and time available per MHR $(T A)$ is calculated and with the division of $T R$ and $T A$, the required number of MHRs is determined. 


\section{The particle swarm optimization algorithm}

The particle swarm optimization (PSO) algorithm search is implemented for the optimum space in a multidimensional complex space. The population of particles moves at some initial velocity in the multidimensional complex space and during the search phase, the algorithm socially interacts with a particle out of the population of particles moving in the multidimensional search area. The algorithm communicates among the moving particles. A unit particle is with some initial velocity and an initial position. The increment in the position of each unit particle is dependent on the velocity of the particle, which is also considered as the best global position of a particle in a multi-dimensional search area (Kumar \& Sridharan, 2010). Initially, the particle's population is at random positions $p(t)$ and velocities vi(t), after which the fitness function is checked. During the algorithm's iteration process, the position and velocity of each unit particle are revised and updated. The fitness function is checked in the iteration and the value of the fitness function is evaluated against the new resulting yield of the fitness function and also with the new position and new velocity gained in the next iteration. The iteration in PSO algorithm is carried out by Eq. (10).

In the exploration process, the particle's new position (also referred to the new solution) pi(t), is compared with its previous positions (also referred to previous solutions). In comparison, if a new solution is found to be better than the old solutions then the algorithm stores the new solution value and updates it as the particle's best position i.e. $p_{\text {ibest. }}$ The global best position $\left(p_{\text {gbest }}\right)$ is the particle's best position in the whole population of the particles in the whole multidimensional search space which is also stored by the algorithm. The particles in the multidimensional search area keep on changing and further update their velocities and position, till the conditions of the best velocity and position are achieved according to the termination criteria. Eq. (10) describes the process of updating the velocities of the unit particles.

$$
\begin{aligned}
& v_{i}(t+1)=v_{i}(t)+\left(c_{1} \times \operatorname{rand}() \times\left(p_{i}^{\text {best }}-p_{i}(t)\right)\right)+\left(c_{2} \times \operatorname{rand}() \times\left(p_{\text {gbest }}-p_{i}(t)\right)\right) \\
& p_{i}(t+1)=p_{i}(t)+v_{i}(t)
\end{aligned}
$$

where,

$v i(t+1)=$ velocity update of the ith particle,

$c_{1}$ and $c_{2}=$ weights applied for local and global optimum positions respectively,

$p i(t)=$ position of the $i_{\text {th }}$ particle at a time interval of $t$,

$p_{i \text { best }}=$ local best position of the $i_{\text {th }}$ particle,

$p_{\text {gbest }}=$ best global position.

rand ()$=$ generation of random variable $\in[0,1]$.

Position and velocity of the unit particle are updated by Eq. (10) and search the best position among all local particles at a time interval of $t$. Unit particle's position is updated according to Eq. (11).

In Eq. (10), the first part of the equation represents the velocity part during the previous iteration steps and the second component is referred to the cognitive component which interacts within the present position of the particle and the best position of the particle. The interaction phase between the particles is referred as the social learning phase (Brownlee, 2011). The particle's new position is updated according to Eq. (11).

\subsection{The memetic algorithm}

The term 'meme' is also known as the unit of a system's cultural information and presents the interaction of genetic and cultural evolution together. The memetic procedure is evolved from the interaction of cultural and genetic evolution. Generalization of genes into unit systems is performed by the memetic algorithm. The information is stored in the unit systems and then the unit systems are introduced to the evolutionary forces for further selection and variation (Brownlee, 2011). The 
global search methodology is applied for processing of information which further exploits the population and also search for good population space in a defined search area. The search procedure is kept on repeating in the form of the iteration process until a local optimum solution is yielded. The memetic algorithm has properties of cultural evolution as well as of genetic evolution. The memetic algorithm can also perform processes such as inheritance, selection, transfer, and variation of the memes and genes.

\subsection{The MMPSO algorithm}

The effectiveness and performance of an algorithm can be checked from its capability to carry out the exploration (global search) and the exploitation (local search) of the particles moving in the multidimensional search space. The particles position and velocity are analyzed in a PSO algorithm during the iteration process. The positions of particles converge towards specific points prematurely at their initial stage. In the iteration process of the PSO algorithm, the particles move towards the global best position at a very fast rate which creates a very small opportunity for the exploitation process in comparison to the exploration process. However, an optimum local solution can be yielded from sufficient exploitation of the solutions. The local search capability of the PSO algorithm can be increased by integrating the PSO algorithm with an algorithm possessing good local search capabilities. In this paper, a combination of PSO algorithm for an optimum global search solution and MA algorithm for an optimum local search solution are chosen. The combined algorithm is applied for optimization of MHRs fleet size for three different sizes of the FMS layout. The new proposed algorithm is referred as the modified memetic particle swarm optimization (MMPSO) algorithm. The MMPSO algorithm is found to have good global and also local search capability. The aforesaid procedure combines the search solutions (particle's position) similar to the crossover action performed in the genetic algorithm (GA). The recombination of particles is accomplished on randomly selected $\mathrm{p} \%$ of the population of the particles. It is observed that after recombination of particles the new solutions have improved fitness values in comparison to the previously stored solution. The new solutions with improved fitness values are stored and updated in place of old solutions. The new proposed MMPSO algorithm performs a good global and local search of solutions and brings a balance between exploration process and exploitation process of the algorithm so that optimum resulting yield can be observed. The MMPSO algorithm applied for optimization of MHRs fleet size satisfactorily optimize the results (Tiwari et al., 2011) and the flowchart of MMPSO algorithm is portrayed in Fig. 1.

\section{Experimental results}

The programming of the analytical model and the MMPSO algorithm for estimation and optimization of MHRs fleet size for the three sizes of FMS facilities was carried out in the Matlab software. The three different sizes of FMS layout are portrayed in Fig. 2, Fig. 3 and Fig. 4. The MHR fleet size estimation from the analytical model and from MMPSO algorithm is presented in Table 1 and also shown in the form of a graph in Fig. 5. The FMS layout presented in Fig. 2 consists of 5 work center and one load-unload center, the FMS layout in Fig. 3 comprises of 7 work centers and one load-unload center and the FMS layout shown in Fig. 4 constitutes of 9 work centers and one loadunload center. 


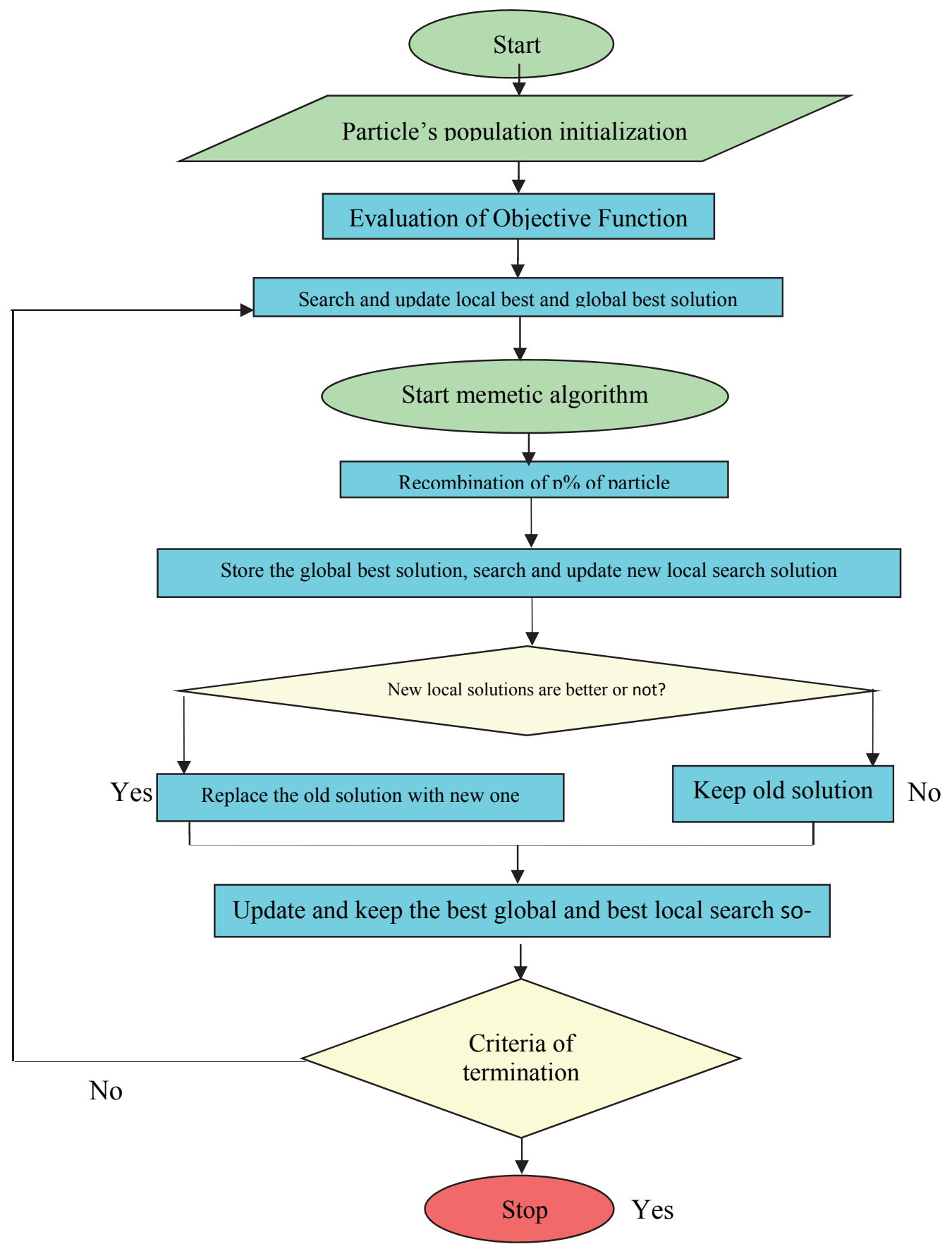

Fig. 1. The Modified Memetic Particle Swarm Optimization Process Flowchart.

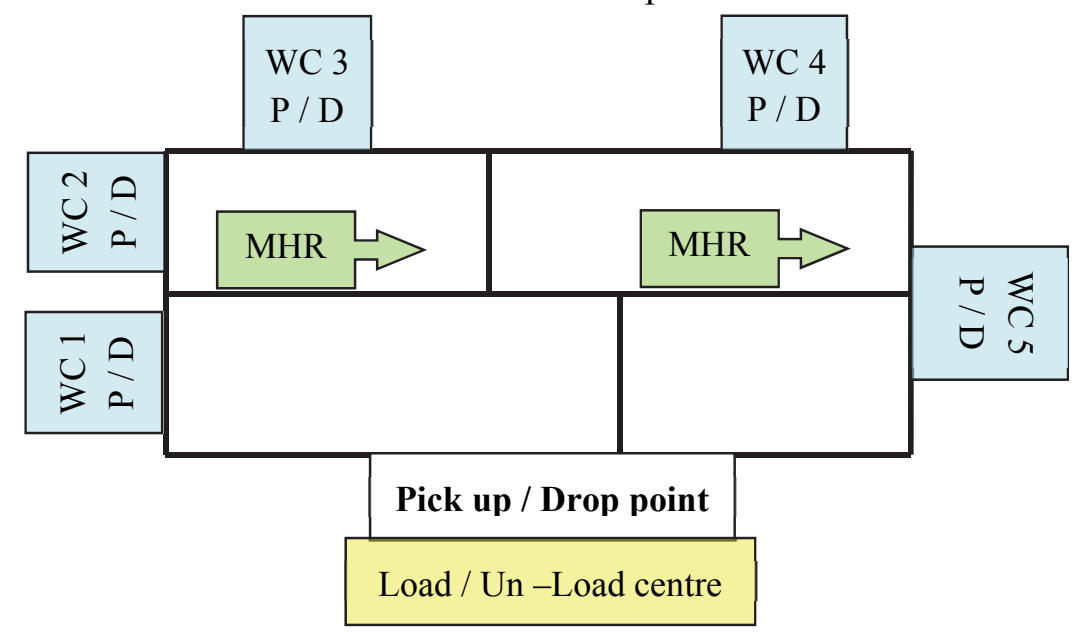

Fig. 2. The FMS layout 1 consisting of 5 work center and one load- unload center 


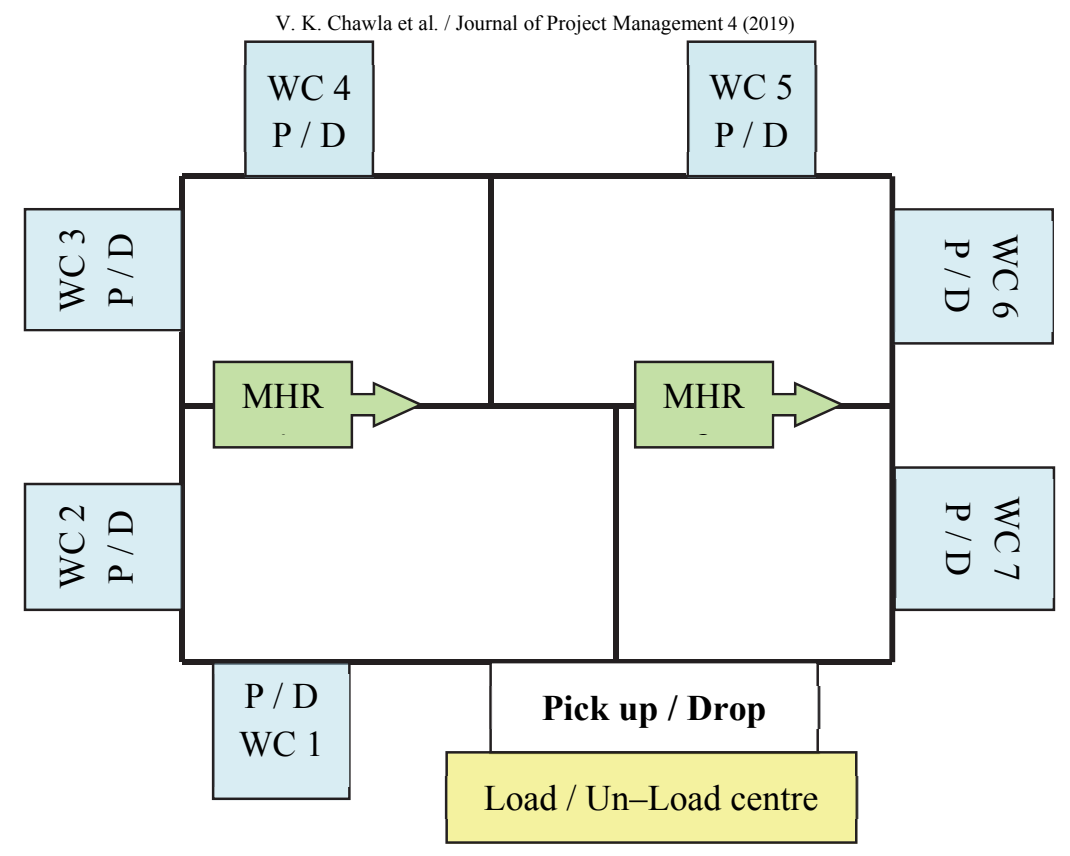

Fig. 3. The FMS layout 2 consisting of 7 work center and one load-unload center.

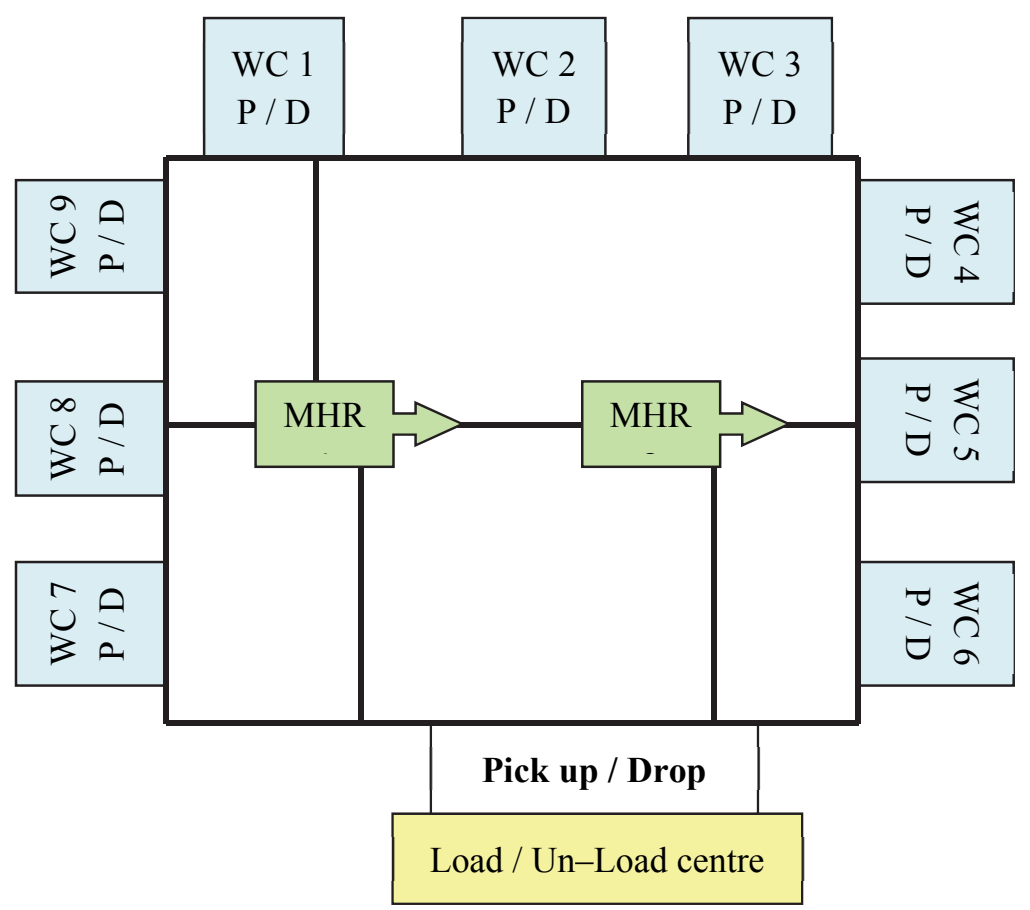

Fig. 4: The FMS layout 3 constituting 9 work center and one load-unload center.

The analytical model and MMPSO algorithm were programmed in the Matlab software and simulated on an Intel(R) Core(TM) i5 processor. During the simulation parameters were tuned as, $\mathrm{c}_{1}=$ $\mathrm{c}_{2}=2.02$, the starting temperature, $\mathrm{F}_{\mathrm{o}}=2.0$, the cooling rate, $\lambda=0.70$. The algorithm was run for 200 iterations, the results from the analytical model for the FMS facility layout 1, 2 and 3 working with 5,7 and 9 work centers and one load - unload center yields 2, 4 and 5 number of MHRs fleet size requirement respectively. Further application of the MMPSO algorithm optimizes the MHRs fleet size to 1, 3 and 4 numbers for the FMS layouts 1,2 and 3 respectively. 
Table 1

MHRs fleet size by the MMPSO algorithm and the analytical model.

\begin{tabular}{|c|c|c|c|c|c|}
\hline \multirow{2}{*}{ FMS layout } & \multirow{2}{*}{ No. of jobs } & \multirow{2}{*}{ No. of Work center } & \multirow{2}{*}{ No. of sequences } & \multicolumn{2}{|c|}{ No. of MHRs } \\
\hline & & & & Analytical & MMPSO \\
\hline 1 & 4 & 5 & 2 & 2 & 1 \\
\hline 2 & 4 & 7 & 2 & 4 & 3 \\
\hline 3 & 4 & 9 & 2 & 5 & 4 \\
\hline
\end{tabular}

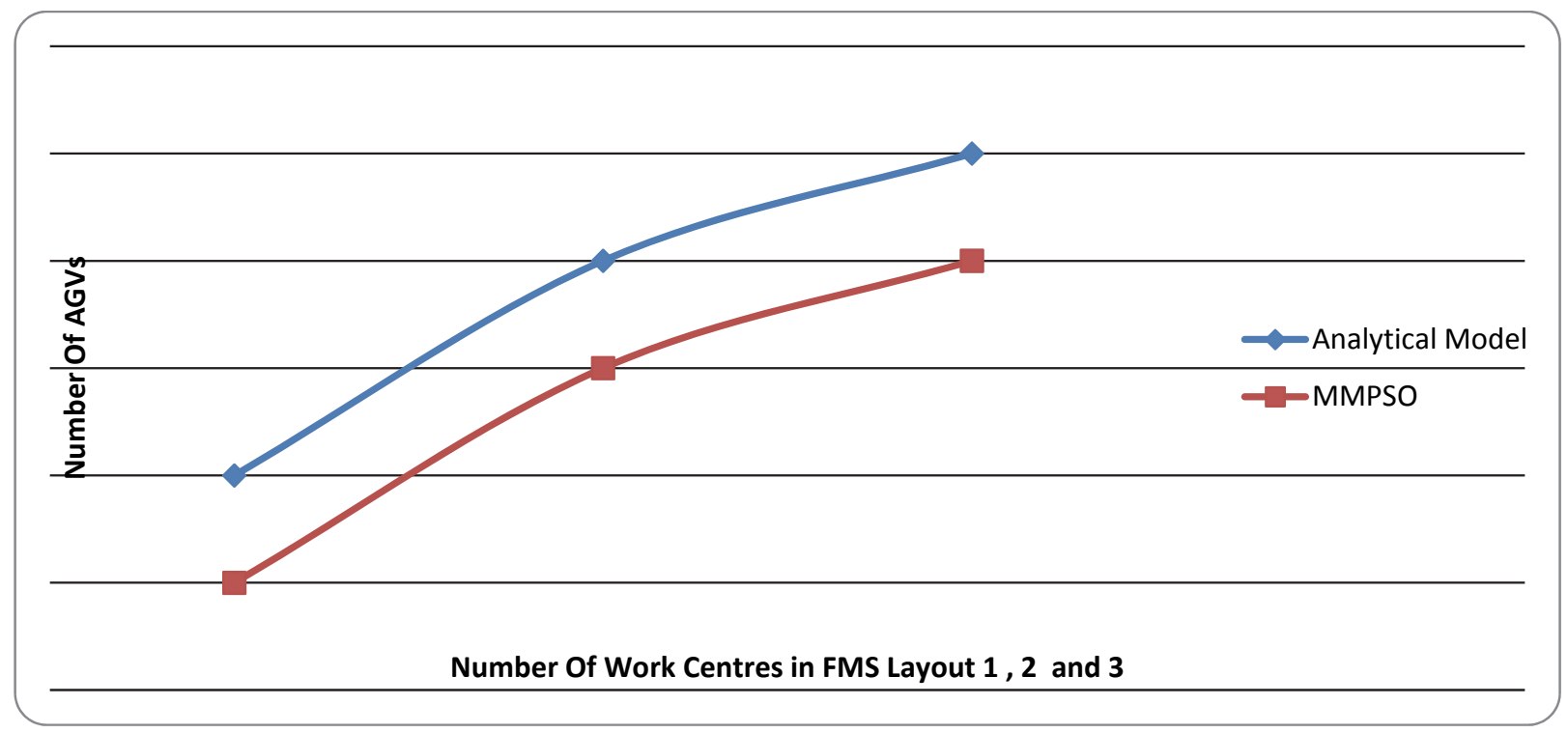

Fig. 5. Comparison of the MHR fleet size by the MMPSO algorithm and the analytical model.

\section{Conclusion and Future Work}

An optimum allocation of resources in the FMS facility is a basic prerequisite for maximum throughput and low makespan. The estimation and optimization of MHRs fleet for three sizes of FMS facility have been presented in this paper. The estimation and optimization of MHRs size were carried out by an analytical model and a new MMPSO algorithm respectively. The results were presented by computational experiment by programming of the analytical model and MMPSO algorithm on the Matlab software. Three different sizes of FMS facility consisted of 5, 7 and 9 work centers and a load-unload center. For an optimum estimate of MHRs fleet size following factors were considered initially in the analytical model namely number of jobs, job processing sequence, number of the work center, total time consumption, job volume mix, job production time, average time, etc. The MMPSO algorithm has been observed to outperform the results of the analytical model and optimizes MHRs fleet size for all three sizes of FMS layout significantly. The MMPSO algorithm optimizes the MHRs fleet size to $50 \%, 25 \%$ and $10 \%$ for the FMS layouts 1, 2 and 3 respectively. It has been also observed that with an increase in a number of work centers in the FMS layouts the difference between the output of the analytical model and MMPSO algorithm also reduces.

The future research paths can be paved for the optimization of MHRs fleet size for the MHRs operating under different dispatching and scheduling policies by application of different evolutionary algorithms such as artificial immune systems, NSGA II or other nature-inspired algorithms. Factors such as reliability of FMS work center, the reliability of MHRs, type of FMS layout, type of MHRs, cruising speed of MHRs, etc. can also be considered for a more accurate and real-time optimization. 


\section{References}

Arifin, R., \& Egbelu, P. J. (2000). Determination of vehicle requirements in automated guided vehicle systems: a statistical approach. Production Planning \& Control, 11(3), 258-270.

Angra, S., Chanda, A., \& Chawla, V. (2018). Comparison and evaluation of job selection dispatching rules for integrated scheduling of multi-load automatic guided vehicles serving in variable sized flexible manufacturing system layouts: A simulation study. Management Science Letters, 8(4), 187-200.

Brownlee, J. (2011). Clever algorithms: nature-inspired programming recipes. Jason Brownlee.

Chanda, A., Angra, S., \& Chawla, V. (2018). A Modified Memetic Particle Swarm Optimization Algorithm for Sustainable Multi-objective Scheduling of Automatic Guided Vehicles in a Flexible Manufacturing System. International Journal of Computer Aided Manufacturing, 4(1), 3347.

Chang, K. H., Huang, Y. H., \& Yang, S. P. (2014). Vehicle fleet sizing for automated material handling systems to minimize cost subject to time constraints. IIE Transactions, 46(3), 301-312.

Chawla, V. K., Chanda, A. K., \& Angra, S. (2017). Evaluation of Dispatching Rules for Integrated Scheduling of MHRs in FMS. In National Conference on Recent Advances in Mechanical Engineering (NCRAME) (pp. 37-41).

Chawla, V., Chanda, A., \& Angra, S. (2018a). Scheduling of multi-load MHRs in FMS by modified memetic particle swarm optimization algorithm. Journal of Project Management, 3(1), 39-54.

Chawla, V., Chanda, A., \& Angra, S. (2018b). Automatic guided vehicles fleet size optimization for flexible manufacturing system by grey wolf optimization algorithm. Management Science Letters, 8(2), 79-90.

Chawla, V., Chanda, A., Angra, S., \& Chawla, G. (2018c). The sustainable project management: A review and future possibilities. Journal of Project Management, 3(3), 157-170.

Chawla, V.K., Chanda, A., \& Angra, S. (2018d). A clonal selection algorithm for minimizing distance travel \& back-tracking of automatic guided vehicles in flexible manufacturing system. Journal of The Institution of Engineers (India): Series C, DOI: 10.1007/s40032-018-0447-5.

Chawla, V., Chanda, A., \& Angra, S. (2018e). Sustainable multi-objective scheduling for automatic guided vehicle and flexible manufacturing system by a grey wolf optimization algorithm. International Journal of Data and Network Science, 2(1), 27-40.

Chawla, V. K., Chanda, A. K., Angra, S., Rani, S., (2018f). Simultaneous Dispatching and Scheduling of Multi-Load MHRs in FMS-A Simulation Study. Materials Today: Proceedings, 5(11), 25358-25367.

Chawla, V. K., Chanda, A. K., \& Angra, S. (2018g). Multi-load MHRs scheduling by application of modified memetic particle swarm optimization algorithm. Journal of the Brazilian Society of Mechanical Sciences and Engineering, 40(9), 436.

Chawla, V., Chanda, A., \& Angra, S. (2019). The scheduling of automatic guided vehicles for the workload balancing and travel time minimi-zation in the flexible manufacturing system by the nature-inspired algorithm. Journal of Project Management, 4(1), 19-30.

Choobineh, F. F., Asef-Vaziri, A., \& Huang, X. (2012). Fleet sizing of automated guided vehicles: a linear programming approach based on closed queuing networks. International Journal of Production Research, 50(12), 3222-3235.

Egbelu, P. J., \& Tanchoco, J. M. A. (1986). Potentials for bi-directional guide-path for automated guided vehicle-based systems. International Journal of Production Research, 24(5), 1075-1097.

Fan, X., He, Q., \& Zhang, Y. (2015). Zone design of tandem loop MHRs path with hybrid algorithm. IFAC-Papers on Line, 48(3), 869-874.

Ganesharajah, T., Hall, N. G., \& Sriskandarajah, C. (1998). Design and operational issues in MHRserved manufacturing systems. Annals of Operations Research, 76, 109-154.

Huang, C. J., Chang, K. H., \& Lin, J. T. (2012). Optimal vehicle allocation for an automated materials handling system using simulation optimization. International Journal of Production Research, 50(20), 5734-5746.

Ji, M., \& Xia, J. (2010). Analysis of vehicle requirements in a general automated guided vehicle system based transportation system. Computers \& Industrial Engineering, 59(4), 544-551. 
Kumar, N. S., \& Sridharan, R. (2010). Simulation-based metamodels for the analysis of scheduling decisions in a flexible manufacturing system operating in a tool-sharing environment. The International Journal of Advanced Manufacturing Technology, 51(1-4), 341-355.

Lin, J. T., Chang, K. H., \& Huang, C. J. (2010, October). Dynamic vehicle allocation in Automated Material Handling System. In Industrial Engineering and Engineering Management (IE\&EM), 2010 IEEE 17Th International Conference on (pp. 1523-1527). IEEE.

Mahadevan, B., \& T. T. Narendran. (1990). Design of an automated guided vehicle-based material handling system for a flexible manufacturing system. The International Journal of Production Research 28(9), 1611-1622.

Mahadevan, B., \& Narendran, T. T. (1994). A hybrid modeling approach to the design of an MHRbased material handling system for an FMS. The International Journal of Production Research, 32(9), 2015-2030.

Maxwell, W. L., \& Muckstadt, J. A. (1982). Design of automatic guided vehicle systems. IIE Transactions, 14(2), 114-124.

Moghadam, B. F., Sadjadi, S. J., \& Seyedhosseini, S. M. (2010). An empirical analysis on robust vehicle routing problem: a case study on drug industry. International Journal of Logistics Systems and Management, 7(4), 507-518.

Moghaddam, B. F., Ruiz, R., \& Sadjadi, S. J. (2012). Vehicle routing problem with uncertain demands: An advanced particle swarm algorithm. Computers \& Industrial Engineering, 62(1), 306-317.

Singh, R., \& Khan, B. (2016). Meta-hierarchical-heuristic-mathematical-model of loading problems in flexible manufacturing system for development of an intelligent approach. International Journal of Industrial Engineering Computations, 7(2), 177-190.

Sinriech, D., \& Tanchoco, J. M. A. (1992). Impact of empty vehicle flow on the performance of single-loop MHR systems. The International Journal of Production Research, 30(10), $2237-$ 2252.

Srinivasan, M. M., Bozer, Y. A., \& Cho, M. (1994). Trip-based material handling systems: throughput capacity analysis. IIE Transactions, 26(1), 70-89.

Tanchoco, J. M. A., Egbelu, P. J., \& Taghaboni, F. (1987). Determination of the total number of vehicles in an MHR-based material transport system. Material flow, 4(1-2), 33-51.

Tiwari, M., \& Harding, J. A. (2011). Evolutionary computing in advanced manufacturing (Vol. 73). John Wiley \& Sons.

Tompkins, J. A., \& White, J. A. (1984). Facility planning. New York: Wiley.

Van der Meer, R. (2000). Operational control of internal transport (No. TTS; T2000/5).

Vis, I. F., De Koster, R. M. B. M., Roodbergen, K. J., \& Peeters, L. W. (2001). Determination of the number of automated guided vehicles required at a semi-automated container terminal. Journal of the Operational research Society, 52(4), 409-417.

Vivaldini, K., Rocha, L. F., Martarelli, N. J., Becker, M., \& Moreira, A. P. (2016). Integrated tasks assignment and routing for the estimation of the optimal number of MHRS. The International Journal of Advanced Manufacturing Technology, 82(1-4), 719-736.

Yifei, T., Junruo, C., Meihong, L., Xianxi, L., \& Yali, F. (2010, July). An estimate and simulation approach to determining the automated guided vehicle fleet size in FMS. In Computer Science and Information Technology (ICCSIT), 2010 3rd IEEE International Conference on (Vol. 9, pp. 432-435). IEEE.

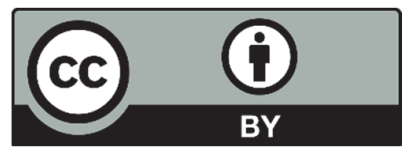

(C) 2018 by the authors; licensee Growing Science, Canada. This is an open access article distributed under the terms and conditions of the Creative Commons Attribution (CC-BY) license (http://creativecommons.org/licenses/by/4.0/). 